\title{
A waveguide coupled LED on SOI by heterogeneous integration of InP-based membranes
}

\author{
A. De Groote ${ }^{1,2, *}$, P. Cardile ${ }^{3}$, A.Z. Subramanian ${ }^{1,2}$, M. Tassaert ${ }^{1,2}$, D. Delbeke ${ }^{1,2}$, R. Baets ${ }^{1,2}$, G. Roelkens ${ }^{1,2}$ \\ ${ }^{1}$ Photonics Research Group, Department of Information Technology, Ghent University - imec, Ghent, Belgium \\ ${ }^{2}$ Center for Nano- and Biophotonics (NB-Photonics), Ghent University, Ghent, Belgium \\ ${ }^{3}$ Center for Microsystems Technology, Ghent University - IMEC, Ghent, Belgium \\ *Email: andreasdegroote@intec.ugent.be
}

\begin{abstract}
A novel design geometry for an optically pumped LED by integrating thin III-V membranes on SOI is proposed. Simulation results predict power efficiency and fabrication tolerance. We present a first proof-of-concept demonstration of the LED.
\end{abstract}

\section{INTRODUCTION}

Though initially aimed at data and telecom applications, silicon photonics is rapidly branching out to a broader range of applications, such as sensing and microscopy. For spectroscopic sensing applications often a broadband optical source is required. Generally speaking, one can choose between three options for the optical source: tunable lasers, laser arrays and (superluminescent) LEDs. Since the realization of light sources in silicon is very difficult, heterogeneous integration of III-V materials such as InP with the SOI platform has been developed. However, designs such as in [1] and [2] still require many processing steps after bonding. Of the three options above, only LEDs are simple enough to achieve decent yield, but they will typically suffer from poor efficiency.

Therefore we propose a novel device geometry. By integrating thin InP-based membranes, we can create an efficient, optically pumped LED. The III-V processing after bonding is straightforward. In order to have a fully integrated device, a pump light source such as a VCSEL can be envisaged to be flip-chip bonded on top of an SOI grating coupler [3], [4].

\section{DEVICE DESIGN}

The overall device design is illustrated in figure 1. It consists of a SOI photonic wire, a III-V ridge waveguide and a tapering section connecting the two. The SOI photonic wire is a fully etched $220 \mathrm{~nm}$ thick silicon-on-insulator wire. Because of the adhesive bonding process used, the upper cladding is DVS-BCB. The III-V ridge waveguide is composed of a InP-based 120nm thin membrane, consisting of multiple InGaAsP quantum wells and barriers and two InP SCH layers. The ridge etch goes through the upper SCH and active region. The dimensions and shape of this membrane were chosen such that

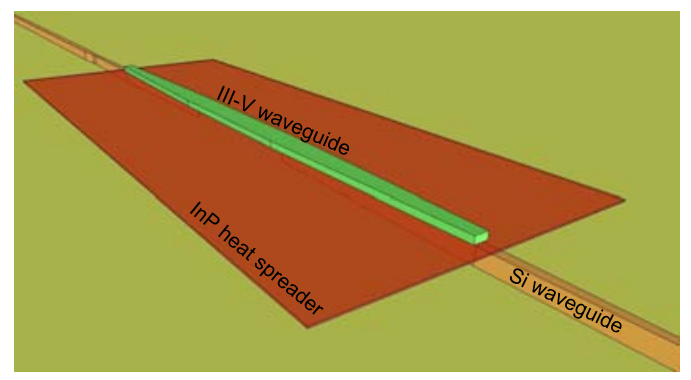

Fig. 1: Illustration of the proposed LED structure.

the optical pump (injected through the silicon waveguide) will be efficiently absorbed and the spontaneous emission will be efficiently collected. A ridge waveguide is preferred over a stripe because of thermal reasons as the lower SCH acts as a heat spreader. Because of the high index contrast in the III-V membrane waveguide, the tapering section can be short $(18 \mu \mathrm{m})$ and fabrication tolerant. We elaborate on the choice of these parameters in the sections below.

The proposed LED device structure can be operated either in reflection or in transmission. Given the strong pump absorption, the carrier concentration will be highest at the incoming taper and lower towards the end of the III-V waveguide due to pump depletion. For the signal in transmission, there will be an optimum length as a very long device may not be pumped completely to transparency and hence absorb the signal. A signal in reflection does not suffer from this drawback, but adds more complexity to the SOI design as one needs a circuit to (de)multiplex the pump and signal.

\section{A. Efficient pump absorption}

A high confinement in the multi-quantum well region is of key importance for efficient pump absorption. Figure 2 shows the confinement in each quantum well as a function of the membrane thickness. The quantum wells and the $\mathrm{SCH}$ layer were chosen to be $8 \mathrm{~nm}$ and $20 \mathrm{~nm}$ thick respectively, the barrier thickness varries. The simulations were carried out with FIMMWAVE, a commercial eigenmode solver (by Photon Design). We assumed a pump at $1310 \mathrm{~nm}$ (TE mode), a ridge width of $1.5 \mu \mathrm{m}$ and varied the number of quantum wells from 1 to 4 . Our chosen thickness of $120 \mathrm{~nm}$ is indicated by the black vertical line.

One can see that there is a clear optimum as a function of membrane thickness. The optimum is at $115 \mathrm{~nm}$ and the confinement

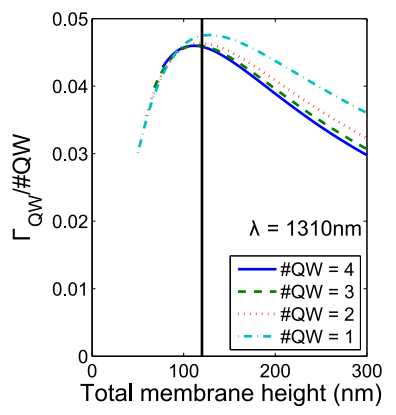

Fig. 2: Confinement as a function of III-V membrane thickness.

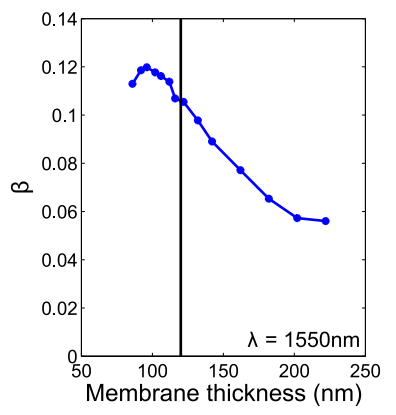

Fig. 3: Spontaneous emission collection efficiency as a function of III-V membrane thickness. 
per well is virtually independent of the number of quantum wells. Hence, we are best to put as many quantum wells in the $115 \mathrm{~nm}$ as possible. We choose 4 quantum wells, which give us a total confinement of $18.4 \%$.

\section{B. Efficient collection of spontaneous emission}

The high refractive index contrast also benefits the collection of the generated spontaneous emission. This effect was simulated with Lumerical FDTD Solution, where the coupling of a dipole source (1550nm, TE polarization) in the quantum wells to the fundamental TE mode is calculated. Various dipole positions were assumed, based on the results from figure 2. Figure 3 shows the collection efficiency $\beta$ as a function of the membrane thickness. There is again a clear optimum, this time at about $96 \mathrm{~nm}$, where $12 \%$ of the radiated light is collected in one direction (there is another $12 \%$ collected in the reverse propagating mode). At our membrane thickness the collection is slightly lower, at $10 \%$.

\section{Thermal heatsink}

To simulate the effect of heat on this small device, we used COMSOL's heat transfer module. We assume the active region to be a source of heat, the entire membrane is clad in DVS-BCB, $320 \mathrm{~nm}$ below and $2 \mu \mathrm{m}$ on top. The buried oxide is $2 \mu \mathrm{m}$ thick, with the silicon substrate at room temperature. A stripe waveguide of $1.5 \mu \mathrm{m}$ wide and $120 \mathrm{~nm}$ high has a length specific heat resistance of $76 \mathrm{KcmW}^{-1}$. For the same rib waveguide, where the $20 \mathrm{~nm}$ bottom InP $\mathrm{SCH}$ is $40 \mu \mathrm{m}$ wide, the length specific heat resistance has reduced to $45 \mathrm{KcmW}^{-1}$. Hence, although the $20 \mathrm{~nm}$ thick InP heat spreader has virtually no influence on the optical mode, the thermal resistance has been decreased by $40 \%$.

\section{Efficient and simple tapering section}

Because of the limited thickness of the III-V membrane, the effective refractive index is only 2.3 (for a $1.5 \mu \mathrm{m}$ wide device). This means that even with a $220 \mathrm{~nm}$ thick silicon device layer, we can make an efficient, fabrication tolerant taper design. This is confirmed with the FIMMPROP module of FIMMWAVE. The width of the III-V taper tip was varied for various bonding BCB thicknesses. Figure $4 \mathrm{a}$ shows the transmission when the silicon waveguide is $220 \mathrm{~nm}$ high and $700 \mathrm{~nm}$ wide. The black vertical line indicates a III-V width of $800 \mathrm{~nm}$, which can be well defined with i-line contact lithography. The maximum loss is less then $0.5 \mathrm{~dB}$.

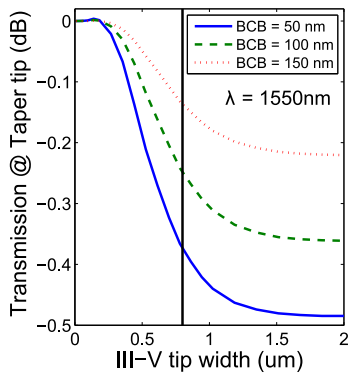

(a)

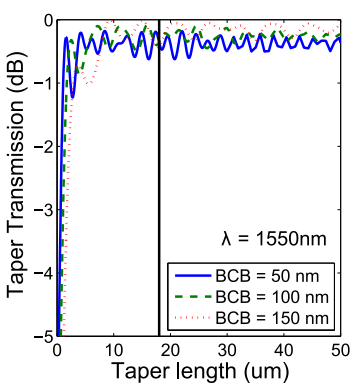

(b)
Fig. 4: (a) Transmission through taper tip as a function of the III-V tip width. (b) Taper transmission as a function of taper length.

The tapering section itself is composed of a double taper, with the silicon and III-V tapering at the same time. Figure $4 \mathrm{~b}$ shows the taper transmission as a function of the tapering length for various $\mathrm{BCB}$ bonding layer thicknesses. For the longer taper lengths, we can see that the transmission is quasi constant. This indicates that indeed our abrupt interfaces such as the taper tips don't induce a lot of loss or higher order modes. We see that the transmission for the thicker bonding layers is slightly better, which is as expected from figure $4 \mathrm{a}$. The difference is within $0.5 \mathrm{~dB}$ though. The shorter tapers are of course not adiabatic, which can clearly be seen. Once the taper is longer then $\pm 10 \mu \mathrm{m}$, we come into the adiabatic regime. This means that we can make very short tapers, even as short as $18 \mu \mathrm{m}$ is a safe choice.

\section{EXPERIMENTAL RESULT}

The depicted device was fabricated using wet etching and photoresist masks. The SOI consisted of a $2 \mu \mathrm{m}$ thick burried oxide and a $220 \mathrm{~nm}$ silicon layer. The InP based membrane has two $20 \mathrm{~nm}$ thick InP $\mathrm{SCH}$ layer with 4 sets of InGaAs-InGaAsP quantum well and barriers in between. The bonding BCB layer was 70nm thick. A SEM image of the fabricated device can be found in figure 5. Figure 6 shows the spontaneous emission spectrum in the silicon waveguide. The $50 \mu \mathrm{m}$ long LED was pumped using a $1310 \mathrm{~nm}$ laser diode through the waveguide $(0.5 \mathrm{~mW}$ in the $\mathrm{Si}$ waveguide) and the generated light was measured both in reflection and transmission. We can see that the
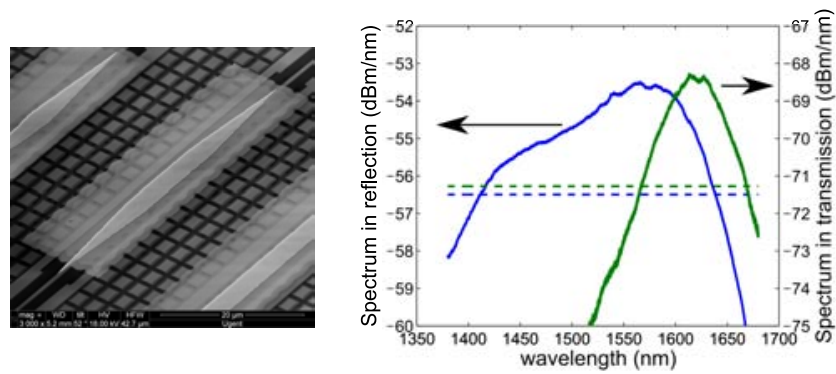

Fig. 5: SEM top view of Fig. 6: Measured spectrum from the fabrication sample. LED light coupled to the silicon waveguide

signal power spectrum in transmission is $\pm 15 \mathrm{~dB}$ lower than the one in reflection. Also the peak wavelength and the $3 \mathrm{~dB}$ bandwidth are different: for the signal in reflection, these are $1565 \mathrm{~nm}$ and $227 \mathrm{~nm}$ respectively, in transmission $1613 \mathrm{~nm}$ and $100 \mathrm{~nm}$. Note that these bandwidths are huge, the reason is currently under investigation. The fact that the signal in transmission is blue shifted with respect to the one in reflection could indicate the LED is not pumped completely. A similar spectrum is generated at the beginning of the LED, but towards the end the shorter wavelengths are absorbed again.

\section{SUMMARY}

To combine easy fabrication and efficiency, an optically pumped membrane LED was proposed and supported by simulations. A first experimental demonstration of the optically pumped LED in both reflective and transmissive mode was also shown.

\section{REFERENCES}

[1] G. Roelkens et al., "III-V/silicon photonics for on-chip and intra-chip optical interconnects," Laser \& Photonics Reviews, vol. 4, no. 6, 2010.

[2] A. De Groote et al., "Heterogeneously integrated iii-v-on-silicon multibandgap superluminescent light-emitting diode with $290 \mathrm{~nm}$ optical bandwidth," Optics letters, vol. 39, no. 16, pp. 4784-4787, 2014.

[3] M. Tassaert et al., "Bias-free, low power and optically driven membrane inp switch on soi for remotely configurable photonic packet switches," Optics express, vol. 19, no. 26, pp. B817-B824, 2011.

[4] J. Schrauwen et al., "Polymer wedge for perfectly vertical light coupling to silicon," in SPIE OPTO: Integrated Optoelectronic Devices. International Society for Optics and Photonics, 2009, pp. 72 180B-72 180B. 